\title{
1. Introduction, brief history, topics addressed, how to use this book, and glossary of terms
}

We propose 'conversational storytelling interviewing' (CSI) as an alternative to semi-structured interviewing. Within 'storytelling science', conversational storytelling is part of a research methodology called 'self-correcting induction'.

In this book, we will explore how induction is part of the 'self-correcting' triad known as 'Abduction-Induction-Deduction' (AID) and is not just induction alone. Abduction is an intuitive, 'hunch' type of hypothesis, to which we then apply Induction tests of sampled cases so that we can make statements about a population. Deduction starts with theory, then draws hypotheses from theory, then tests the hypotheses by using data and cases.

By using all three of these AID forms of inquiry, the research reflects back on itself and allows for corrections and attunements at each stage. This is why AID is called a 'self-correcting' method. In some ways, 'self-correcting' is like a revival of formerly popular 'pilot studies', here more formalized and integrated as part of the storytelling science research process. This book will help you to both understand and apply these AID and self-correcting methods in your own research.

\section{HISTORY}

The classic Hawthorne Studies were transformed when, in July of 1929, after 1,600 interviews, they halted the project, and changed their interviewing method away from semi-structured and structured interviews. They replaced the 'direct approach to questioning' with the new 'indirect approach' in which people told their accounts and stories in conversations, without interruption, without the interviewer trying to herd the (storyteller) back to some a priori topics and sub-topics (Roethlisberger et al. 1939: 203). This indirect approach is what we (David and Grace Ann, in this book) now call 'conversational storytelling interviewing' (CSI). This indirect method was found to produce fewer social desirability effects. Instead of semi-structured interviews, the Hawthorne psychologists and psychoanalysts recommended a purposive storytelling conversation. 
It is time to move beyond current over-reliance on semi-structured interviewing as a very limited business storytelling and social science methodology. We recommend a shift towards CSI. CSI combines classic 'indirect' storytelling interviewing, and incorporates dialogical and dialectical methods along with the AID 'self-correcting' cycles. This provides a very comprehensive and robust method. CSI also offers a personally meaningful and satisfying experience for both interviewees as well as interviewer researchers, as several successfully defended PhDs can attest.

\section{TOPICS ADDRESSED}

We will begin by answering the question: What is storytelling? We will look at the dialogical as well as dialectical dimensions of conversations. We introduce four (4) types of tests that may be applied in the self-correcting process. Then we offer a case example of using the AID process and the four (4) types of test that can be applied. We offer a 'storytelling paradigm' which positions self-correcting inductions within the context of theories, methodologies, and interventions relevant to 'storytelling science'. We address the entire dissertation research process, from choosing your topic and writing your theory and methods sections, through to managing your oral exams and post-submission job processes.

We discuss both quantitative and qualitative induction. We consider the metaphysics of storytelling science, and address both antenarrative and anti-narrative. We position CSI within the context of other approaches such as Grounded Theory, 4th Wave Grounded Theory, Appreciative Inquiry, and the Socio-Economic Approach to Management (SEAM) (Boje 2016d; Boje and Cai-Hillon 2017).

\section{FORMAT: HOW TO USE THIS BOOK}

This user-friendly book allows both the novice and the experienced storytelling researcher to find value. For the novice there is a helpful Glossary of Terms, organized by topic and also alphabetically indexed. For those more advanced or aspiring to become advanced, in each chapter, selected topics are explored in the 'In Depth' sections. In addition, each chapter has activities and/ or questions for self-reflexivity in the 'Exercise' boxes. Some of these activities may be done alone, some with a partner or partners, and some are suited for groups. All have been used successfully in either workshops or classrooms.

\section{GLOSSARY OF TERMS GROUPED BY TOPIC}

(See below this list for terms organized alphabetically and numbered 1-32.) 


\section{Abduction, Induction, Deduction, Self-correcting Method}

1. Abduction (\#1 below): The ' $A$ ' in the AID (Abduction, Induction, Deduction) process; an intuitive guess, a tentative hypothesis, or speculative insight about what is being studied.

2. Induction (\#16 below): Gather qualitative or quantitative empirical data, and from that data develop generalizations and theories (thus going from observation to theory).

3. Deduction (\#7 below): The process in which a researcher starts with an existing theory, then uses the theory to develop a hypothesis about what is being studied, then tests the hypothesis to support or refute the theory (thus going from theory/hypothesis to testing/observation).

4. Self-Correcting Method (\#23 below): Using Abduction, Induction, and Deduction (AID) together in research. By this self-correcting learning by trial and error using the scientific method, we arrive closer to the truth (Popper 1963: 318).

\section{Abstracting, Grounding}

5. Abstracting (\#2 below): Reducing place, context, and change to universals or generalizations. Abstracting in Western narrative, reduces time to linear (beginning, middle, and end), develops a few events and characters to stand for the whole historical detail, and reduces place to spatial concepts rather than nature and community specificity. Abstracting and 'grounding' are aspects of a vertical axis of storytelling (see grounding).

6. Grounding (\#14 below): Vertical axis of storytelling, 'grounding' is contextual, about specifics of place, its nature, community, and living stories. (See abstracting.)

7. True Storytelling (\#31 below): An ethics approach to arriving closer to truth.

Antenarrative, Six Bs of Antenarrative, Linguistic Turn, Sociomaterialism, Narrative, Storytelling, Storytelling Organization, TamaraLand

8. Antenarrative (\#3 below): What comes before the story is constituted as 'the story'. Processes which are (pre)constitutive of both narratives and stories. Vertical axis antenarrative processes include 'abstracting' (typical of narrative-counternarrative that is often placeless), and 'grounding' in place (characteristic of indigenous living stories). Horizontal axis antenarrative processes include 'futuring' (prospective sensemaking, scenarios), and rehistoricizing (retrospective sensemaking narratives). (See Six Bs of Antenarrative.) 
9. Six Bs of Antenarrative (\#24 below): Before, beneath, bets on the future, becoming, between, and beyond. Before (forehaving), beneath (foreconcepts), bets on the future (foresight), becoming (forecaring), between (forestructuring in advance), and beyond (foregrasping by intuition).

10. Linguistic Turn (\#18 below): In the 1970s, humanities and social sciences made a shift based on the claim of the primary importance of language in meaning-making and narrative. A countermovement, called socio-material, has claimed the linguistic turn went too far in dismissing the material aspects in the ontology of meaning-making. (See Sociomaterialism, Ontology, Narrative.)

11. Sociomaterialism (\#27 below): The entanglement of the social with materiality.

12. Narrative (\#20 below): Since Aristotle (350 BCE), narrative to be coherent has a beginning, middle, and end (linear time), with six elements (in order of importance): plot, characters, theme, dialogue, rhythm, and spectacle. Recently, spectacle has taken first place. Narrative requires story to be '.. a whole ... a whole is that which has beginning, middle, and end' (Aristotle, 350 BCE: 1450b: 25, p. 233). Kenneth Burke (1945) renamed Aristotle's (350 BCE) narrative form elements and reduced them to five in his Pentad version. Act (plot), actor (characters), purpose (theme), agency (dialogue + rhythm), scene (spectacle). 'Typically searching for a causal chain', 'the plot follows - either the sequence beginning-middle-end or the sequence situation-transformation-situation. But sequence is the source of sense' (Weick 1995: 128). Czarniawska (1998: 78), for example, says narrative, or 'a story consists of a plot comprising causally related episodes that culminate in a solution to a problem'. Elsewhere, Czarniawska (1998: 2) clarifies, 'for them to become a narrative, they require a plot, that is, some way to bring them into a meaningful whole'.

13. Storytelling (\#28 below): The general term for the whole field, including Grand Narratives as well as Microstoria, and also including the pre-constitutive elements of storytelling called 'antenarrative' (Boje, 2001).

14. Storytelling Organization (\#29 below): Storytelling organizations are defined as multi-voices, multi-stylistic, multi-medium, and multi-languaged, and out of this heteroglot, storytelling organization can emerge.

15. TamaraLand (\#30 below): Boje's (1995) metaphor for the Storytelling Organization. 'Tamara' was a play by John Krizanc performed in a mansion in Los Angeles. The scenes from the play occurred simultaneously in many rooms, and the audience moved from room to room observing different parts of the story. The audience members never see the 'whole' story at once. Boje says this is what storytelling in organizations is like. 


\section{Auxiliary Assumptions, Fallibilism}

16. Auxiliary Assumptions (\#4 below): Assumptions which underlie our hypothesis and can affect the outcomes of our hypothesis testing. Predictions come not only from a deductive theory on abductive-intuition (or hypothesis), but also come from other theories and assumptions, which are called 'auxiliary assumptions.' Auxiliary assumptions preclude Popper's absolute falsification of a deductive theory or induction-intuition: 'How can one determine whether a theory is falsifiable? The way to do this is to attempt to make predictions from the theory and see if these predictions have the possibility, at least in principle, of being shown to be wrong' (Trafimow 2009: 504).

17. Fallibilism (\#12 below): Error is possible because no theory (deduction), thesis (or antithesis), or abduction (inspiration) can be absolutely empirically proven, since there are always auxiliary assumptions (as well as Kant's citing of transcendental and moral reason issues) that have not been accounted for. (See Auxiliary Assumptions.)

\section{'Big S' Science, 'Little s' Science}

18. 'Big S' science (\#5 below): 'Big $S$ ' science is communal, somewhat bureaucratic evolution of knowledge; 'little s' science is more speculative, more local, and more a 'Native Science' inquiry. Rather than either/or, we contend it is possible to do both/and, both 'big S' and 'little s' science.

19. 'Little s' science (\#19 below): More about local knowing, being, and doing in a situation of place, in IWOK ('Indigenous Ways of Knowing'). (See 'Big S' science, IWOK.)

\section{Conversational Interviewing, Dialogical, Dialectical, Social Heteroglossia}

20. Conversational Interviewing (\#6 below): a back-and-forth exploratory process needed for self-correcting. This back-and-forth can be dialogical, involving sharing and exploring, and it can also be dialectical, with counternarratives (narratives which tell a different story) to the originally expressed narrative.

21. Dialogical (\#8 below): The way that communication between two or more people goes back-and-forth in a non-adversarial way that builds rapport and relationships. It may be confrontative, but typically in a win-win collaborative type of confrontation rather than a win-lose process. There are several dialogisms, the most known is polylogical (many logics), and polyphonic (many voices). Other dialogisms include stylistics (architectural, oral, written texts), chronotopes (nine spacetime relationships in the history 
of the novel), and architectonics (interanimation of cognitive, aesthetic, and ethics discourses).

22. Dialectical (\#9 below): There are two types of dialectic. First, and most known, is the thesis-antithesis-synthesis (TAS) dialectic that tends to dualize into opposite polarized perspectives. TAS is often a dynamic of narrative-counternarrative dialectics. Second is the "negation of the negation' $(\mathrm{N} / \mathrm{N})$ that is more dialogical, with many perspectives.

23. Social Heteroglossia (\#26 below): The dialogical opposition of centripetal (centralization) forces with centrifugal (decentralization) forces of language and ideological-discourse.

\section{Entanglement, Quantum Storytelling, Spacetimemattering}

24. Entanglement (\#10 below): This refers to quantum-ness, where space and time and matter overlap with each other, and are therefore inseparable. (See Spacetimemattering.)

25. Quantum Storytelling (\#22 below): The entanglement of spacetimemattering (their inseparability) in Being (see Ontology), and 'grounding' in place, in nature, and community. (See Grounding.)

26. Spacetimemattering ( $\# \mathbf{2 5}$ below): The quantum view that space and time and matter overlap (are entangled) with each other.

\section{Epistemology, Ontology}

27. Epistemology (\#11 below): How we know what we think we know. Ways of knowing.

28. Ontology (\#21 below): Metaphysics branch focused on 'nature of Being'. Being is usually capitalized to differentiate from beings (living beings). Ways of Being-in-the-world (Heidegger 1927/1962). Spacetimemattering Being-in-the-world. (See spacetimemattering.)

\section{4th Wave Grounded Theory, Grounded Theory}

29. 4th Wave Grounded Theory (\#13 below): This uses AID in self-correcting cycles; starts with Abduction before moving to Induction, then either revises Induction or moves to the Deduction phase of hypothesis testing. Then the cycle may loop back to Abduction, Induction, or Deduction for another cycle.

30. Grounded Theory (\#15 below): An 'inductive' research process that begins with observations and data collection, and from that develops theory (Glaser and Strauss 1967); often used in qualitative research. Grounded Theory has gone through four waves of development. 1st Wave embraces crude induction, and swallows the inductive fallacy whole. 2nd Wave Grounded Theory (Strauss and Corbin 1990; 1994/1998; Corbin and 
Strauss 2007) tries to compensate by turning to empirical coding of the theme-forms into categories (typologies) using (post) positivism. 3rd Wave Grounded Theory (or 'constructivist Grounded Theory') departs from 1st Wave and 2nd Wave Grounded Theory by claiming that both theories and date are not discovered, but are constructed by the researcher in their interactions with the field of scholarship. 4th Wave includes process of self-correcting cycles. (See 4th Wave, Self-Correcting Method.)

\section{IWOK, WWOK}

31. IWOK (\#17 below): 'Indigenous Ways of Knowing', based on indigenous ontology, epistemology, and 'Native Science'; views all plants, animals, and the earth and streams as living beings interconnected with humans. Nature is the source of wisdom for humans. The way to knowledge is to study nature and observe the dynamics of the constant flux and interconnectedness of life.

32. WWOK (\#32 below): Western Ways of Knowing, based on Euro-Western ontology and epistemology; tends to see humans as separate from, and superior to, the rest of life and the planet. The way to knowledge is to immobilize, break apart, experiment, and take a snapshot of phenomena.

\section{CROSS-INDEX OF TERMS ORGANIZED ALPHABETICALLY}

(See above for term definitions.)

1. Abduction: See \#1 above.

2. Abstracting: See \#5 above.

3. Antenarrative: See \#8 above.

4. Auxiliary Assumptions: See \#16 above.

5. 'Big S' science: See \#18 above.

6. Conversational Interviewing: See \#20 above.

7. Deduction: See \#3 above.

8. Dialogical: See \#21 above.

9. Dialectical: See \#22 above.

10. Entanglement: See \#24 above.

11. Epistemology: See \#27 above.

12. Fallibilism: See \#17 above.

13. 4th Wave Grounded Theory: See \#29 above.

14. Grounding: See \#6 above.

15. Grounded Theory: See \#30 above.

16. Induction: See \#2 above.

17. IWOK: See \#31 above. 
18. Linguistic Turn: See \#10 above.

19. 'Little s' science: See \#19 above.

20. Narrative: See \#12 above.

21. Ontology: See \#28 above.

22. Quantum Storytelling: See \#25 above.

23. Self-Correcting Method: See \#4 above.

24. Six Bs of Antenarrative: See \#9 above.

25. Spacetimemattering: See \#26 above.

26. Social Heteroglossia: See \#23 above.

27. Sociomaterialism: See \#11 above.

28. Storytelling: See \#13 above.

29. Storytelling Organizations: See \#14 above.

30. TamaraLand: See \#15 above.

31. True Storytelling: See \#7 above.

32. WWOK: See \#32 above. 